\title{
Guideline-based quality indicators-a systematic comparison of German and international clinical practice guidelines
}

Monika Becker ${ }^{1 *}$ D, Jessica Breuing ${ }^{1}$, Monika Nothacker ${ }^{2}$, Stefanie Deckert ${ }^{3}$, Marie Brombach ${ }^{3}$, Jochen Schmitt $^{3}$, Edmund Neugebauer ${ }^{1,4}$ and Dawid Pieper ${ }^{1}$

\begin{abstract}
Background: Evidence-based clinical practice guidelines (CPGs) are relevant sources for generating quality indicators (Qls). The objective of this study was to compare guideline-based Qls of German and international CPGs and their underlying methodological approaches.

Methods: We conducted systematic searches in the guideline databases of G-I-N (Guidelines International Network) and NGC (National Guideline Clearinghouse) between February and June 2017 to identify international CPGs matching the topics of German evidence-based CPGs $(n=35)$ that report Qls, which were identified in a preceding study. Additionally, we searched the websites of the particular CPG providers for separate documents with regard to Qls. We included evidence-based CPGs which report Qls. Reported Qls, the underlying guideline recommendations, and information on methods of development were extracted. The selection and extraction of CPGs were conducted by one reviewer and checked by another. For each matched pair of CPGs, we assessed whether the suggested Qls matched or were not directly comparable.
\end{abstract}

Results: Twenty-five international CPGs, originating from seven CPG providers in total, met the criteria for inclusion. They matched the topics of 18 German CPGs. This resulted in 30 CPG pairs for the comparison of Qls (some of the international CPGs matched the topic of more than one German (PG). We found 27 Ql pairs with Qls "not different or slightly different", corresponding to 13\% (27 of 212) of the Qls in German CPGs and 16\% (27 of 166) in international CPGs. Only two QI pairs were judged to be "different/inconsistent". For 183 of 212 (86\%) Qls from German CPGs and 137 of $166(83 \%)$ Qls from international CPGs, no direct comparison could be made. An explicit link to one or more guideline recommendations was found for 136 of 152 (89\%) Qls from German CPGs and 82 of 166 (49\%) Qls from international CPGs. Some information on methods for the development of Qls existed for 12 of 18 (67\%) German CPGs and 8 of 25 (32\%) international CPGs.

Conclusions: The majority of QIs in German and international CPGs were not comparable. Various reasons for this are conceivable. More transparent reporting of the underlying methods for generating guideline-based Qls is needed.

Keywords: Guidelines, Quality indicator, Performance measures, Systematic review

\footnotetext{
*Correspondence: monika.becker@uni-wh.de

'Institute for Research in Operative Medicine (IFOM), Department

Evidence-based health services research, Faculty of Health, Department of

Medicine, Witten/Herdecke University, Ostmerheimer Str. 200, Building 38,

51109 Cologne, Germany

Full list of author information is available at the end of the article
}

(c) The Author(s). 2019 Open Access This article is distributed under the terms of the Creative Commons Attribution 4.0 International License (http://creativecommons.org/licenses/by/4.0/), which permits unrestricted use, distribution, and

reproduction in any medium, provided you give appropriate credit to the original author(s) and the source, provide a link to the Creative Commons license, and indicate if changes were made. The Creative Commons Public Domain Dedication waiver (http://creativecommons.org/publicdomain/zero/1.0/) applies to the data made available in this article, unless otherwise stated. 


\section{Background}

Quality measurement and improvement play an important role in the provision of healthcare. For this purpose, quality indicators (QIs) can be used. There is no clear-cut definition of a QI. According to Lawrence and Frede, a QI is a "measurable element of practice performance for which there is evidence or consensus that it can be used to assess the quality, and hence change in the quality, of care provided" [1]. The Joint Commission on Accreditation of Healthcare Organizations (JCAHO) defines QIs as "[...] quantitative measures that can be used to monitor and evaluate the quality of important governance, management, clinical, and support functions that affect patient outcomes" [2]. To be deemed as trustworthy and useful, QIs have to satisfy different criteria, such as relevance, validity, reliability, feasibility, and target group orientation [3-6]. To meet the high methodological requirements on QIs, they should be based on scientific evidence and developed in a systematic and transparent way wherever possible [7, 8].

As evidence-based clinical practice guidelines (CPGs) are designed to reflect current best practice, they are relevant sources for generating QIs [7, 9]. The term "guidelinebased QIs" specifically indicates QIs that are either generated from already available CPGs or coupled with the process of CPG development [10]. Besides assessing the quality of healthcare, these are important tools to assess the implementation of guideline recommendations [1113]. However, the methodological approaches to the development of guideline-based QIs vary considerably [10].

In Germany, the AWMF (German Association of the Scientific Medical Societies) provides a methodological framework for the development of CPGs by the scientific medical societies. The guideline classification scheme of the AWMF differentiates between S1-, S2k-, S2e-, and S3-CPGs depending on the methodological approach [14]. Thus, S1CPGs are based on informal consensus-building. In S2kCPGs, a formal consensus method is applied in a representative panel, and S2e-CPGs include a systematic approach to literature-searching as well as the selection and appraisal of evidence. S3-CPGs comprise the requirements for both S2k-CPGs and S2e-CPGs and thus have the highest methodological standard in Germany. An analysis of the status quo of reported QIs in German S3-CPGs, performed in 2013, identified 34 S3-CPGs which report 394 different QIs (including measures of quality labeled as "quality criteria" or "quality measure") [15]. For example, the German S3CPG "Diagnostics, treatment and follow-up care of malignant ovarian tumors" comprises 12 QIs, one of them concerning counselling by social services (numerator: number of patients with counselling by social services; denominator: all patients with an initial diagnoses of ovarian cancer and treatment in a clinical institution) [16]. A recent update of this analysis with a search up to 2016 (Deckert S, et al: (Wie) erfolgt die Ableitung von Qualitätsindikatoren zur
Messung und Bewertung der Versorgungsqualität im Rahmen von S3-Leitlinien? Eine Übersichtsarbeit, submitted) found 35 current German S3-CPGs which report 372 different QIs. Four German S3-CPGs were developed by the National Program for Disease Management Guidelines (NDMG), 15 by the German Guideline Program in Oncology (GGPO), and 16 by various scientific medical societies. Particularly, the CPGs of the NDMG and GGPO have a broad scope and cover various areas of medical care. For these CPGs, the development of guideline-based QIs is obligatory; the methodology is outlined in the corresponding manuals [11-13].

Although a working group of the Guidelines International Network (G-I-N) recently proposed a set of reporting standards for guideline-based performance measures [17], there is currently no gold standard for the development of guideline-based QIs [10, 18]. Moreover, there is a lack of research into the consistency of guideline-based QIs from different CPGs. Our hypothesis is that in many cases, QIs from German S3-CPGs do not correspond with QIs of international CPGs on related topics.

This study was part of the project "Systematic analysis of the translation of guideline recommendations into quality indicators and development of an evidence- and consensus-based standard", supported by the German Research Association (DFG). Our analysis provided information for another part of the research project, a qualitative study which consisted of structured interviews with developers, methodologists, and users of international guidelines (Bolster $\mathrm{M}$, et al: International experiences in the development of guideline-based quality indicators- a qualitative study, submitted). The intention of both studies was to add information to existing research on methods for the guideline-based development of QIs [10, 17]. The results contribute to a consensus study on standards of the translation of guideline recommendations into quality indicators in Germany.

The objective of this study was to compare guidelinebased QIs of the 35 previously identified German S3-CPGs, as well as their underlying methodological approaches, with those of international CPGs on related topics.

\section{Methods}

The study was aligned with the PRISMA guidelines [19], although it did not fulfil all requirements related to a systematic review. The methods were in accordance with those set out in a previously published protocol [20], with the exception of one eligibility criterion that we added later (see below).

\section{Data sources and the selection of CPGs Eligibility criteria}

International CPGs that met the following criteria were included in the study: 
- QIs are reported.

- The CPG is an evidence-based CPG.

- The topic and recommendations are comparable with those of at least one of the 35 previously identified German S3-CPGs (see Additional file 1).

- The country of CPG development belongs to WHOStratum A [21].

- Date of publication between 2012 and 2017.

- Published in German, English, French, Spanish, Dutch, Norwegian, or Swedish.

- The current full-text version is available at no charge.

- The validity date of the CPG, indicated by the CPG developer, is not exceeded.

In addition to the criteria already mentioned in the protocol, we defined as a basic prerequisite that the document is a CPG with clearly identifiable recommendations.

Whenever QIs were solely reported in a separate document which is not a supplement to the CPG (e.g. evidence or methodological report), they had to be linked explicitly with the particular CPG.

An example for such a separate document containing guideline-based QIs is a document from the website of the National Institute for Health and Care Excellence (NICE): "NICE menu of general practice and clinical commissioning group indicators" [22]. The mentioned NICE-QIs are usually linked with specific CPGs. For example, the NICE indicator NM59 (the percentage of patients with diabetes who have a record of an albumin: creatinine ratio (ACR) test in the preceding 15 months) is linked with the NICE-CPGs NG17 (type 1 diabetes in adults) [23] and NG28 (type 2 diabetes in adults) [24]. Otherwise, we assumed that these QIs are not guidelinebased and excluded the CPG.

Evidence-based CPGs were defined in this analysis as CPGs whose recommendations

- Are based on a systematic literature search

- Are clearly identifiable and assigned with a grade of recommendation (GoR) and/or a level of evidence (LoE)

- Are linked to the references of the underlying evidence.

\section{Literature search}

We conducted systematic searches in the guideline databases of G-I-N and NGC (National Guideline Clearinghouse) between February and June 2017 to identify international CPGs matching the topics of the previously identified German S3-CPGs which report QIs. The search strategies included keywords related to the clinical topics, both as full terms and with appropriate truncations, connected with Boolean operators. For six of the CPGs from the CPG program oncology and for all German S3-CPGs on diabetes, we conducted one combined search each ("carcinoma OR "cancer OR oncolog*; diabet"); for the remaining German S3-CPGs, separate searches were performed (see Additional file 2 for details on search strategies). Furthermore, we crosschecked the reference lists of the German S3-CPGs and the international CPGs eligible for inclusion in the analysis.

In cases we identified international CPGs with eligible topics that comprised neither QIs nor links to QIs, we searched the websites of the particular CPG providers for separate documents describing QIs that were explicitly linked with the particular CPG.

\section{Selection process}

One reviewer screened the titles of records. The full texts of those deemed eligible for inclusion were retrieved. Subsequently, full texts were screened by one reviewer and checked by another. The reasons for exclusion were documented, and any disagreements were resolved through discussion and consensus.

In cases where no eligible international CPG matching the topic of a German S3-CPG could be found, we excluded that German S3-CPG from the analysis.

\section{Data extraction}

A standardized data extraction form was developed based on the items used in a previous project on the evaluation of QIs reported in German S3-CPGs [15] and then piloted. For each included matched CPG pair, we extracted only QIs on clinical topics (e.g. screening, diagnostics, therapy, or rehabilitation) that were addressed in both CPGs. For example, if only one of the matched CPGs dealt with the clinical topic "diagnostics", we did not consider QIs on that topic. Furthermore, we collected the following information:

- Number of members and expertise of the QI development group (such as methodologists, clinicians, patient representatives)

- Label of the quality measure, e.g. QI, quality criteria and performance measure

- Categorization of QI into structure, process, or outcome indicators according to the definition of Donabedian [25] (in case of missing assignment by the guideline authors, our own assignment was made)

- Underlying recommendations and whether the QIs were based explicitly or implicitly on those

- Rationale reported for the QI

- Scientific measurement properties reported for the QI, e.g. reliability and validity [26] 
- Intended purpose reported for the QI, e.g. quality reporting, quality management systems, and evaluation of CPGs

- Quality objectives reported

- Methods used for QI development, e.g. search for existing QIs, consensus methods, and assessment tools

Data were extracted by one reviewer and checked by another, and any disagreements were resolved through discussion and consensus.

\section{Quality appraisal}

As trustworthy guideline-based QIs should be based on high-quality CPGs $[10,17]$, we appraised the methodological quality of all included German S3- and international CPGs using the domain "Methodological Rigor of Guideline Development" of the German Instrument for Methodological Guideline Appraisal (DELBI) [27]. Seven items were rated on a 4-point scale (wherein one $=$ "strongly disagree", two $=$ "disagree", three $=$ " agree", and four = "strongly agree"):

- Systematic methods were used to search for evidence.

- The criteria for selecting the evidence are clearly described.

- The methods used for formulating the recommendations are clearly described.

- Health benefits, side effects, and risks have been considered in formulating the recommendations.

- There is an explicit link between the recommendations and the supporting evidence.

- The guideline has been externally reviewed by experts prior to its publication.

- A procedure for updating the guideline is provided.

Two reviewers performed quality assessment independently. In case the appraisal of the two reviewers differed by two or more points, disagreements were resolved through discussion and consensus. The domain score was calculated by summing up the scores of individual items and by standardizing the total as a percentage of the maximum possible score for the domain (4 (strongly agree) $\times 7$ (items) $\times 2$ (appraisers) $)$ [27] .

In case reviewers had been involved in the development of an included CPG, they did not participate in their quality assessment.

\section{Data synthesis}

Data synthesis involved a descriptive analysis and a tabular comparison of the QIs of the international and German S3-CPGs for each clinical topic and, where applicable, for each underlying recommendation. We collected the number of CPGs that provided information on the QI development group, methods of QI development, as well as the rationale and intended purpose of QIs. On the basis of reported QIs, we collected the number of QIs for which quality objectives and measurement properties were reported as well as the number of QIs that were explicitly or implicitly based on guideline recommendations.

For each matched pair of CPGs, we compared the suggested QIs and assessed whether the QIs matched or not.

Our definition of QI-matching was that both QIs on the same clinical topic either agreed or disagreed in content and definitions regarding a specific clinical issue, e.g. a specific intervention or diagnostic procedure and either addressed or did not address the same population. Then, we assigned QIs either to the category "not different/ slightly different" or "different/inconsistent". QIs were considered not to match whenever no direct comparison could be made because the QIs differed fundamentally in contents and definitions. Thus, the QIs either addressed different specific issues within a clinical topic, or were reported in only one of the matched CPGs, even though both CPGs addressed that particular clinical topic. For example, the topic "screening" was addressed by both CPGs of a matched pair, but only one had defined QIs for that topic. Those QIs were extracted under the category "QI only defined in the international or the German S3-CPG", respectively. For each of the categories described above, we collected the number of QIs or QI pairs. The assignment of the QIs to the categories was conducted by one reviewer and checked by another. Disagreements were resolved through discussion and consensus. Furthermore, the methods described for QI development were presented as a narrative summary.

\section{Results}

\section{Results of the literature search and characteristics of included CPGs}

The searches in the CPG databases identified 4889 records. We found seven additional potential eligible international CPGs by crosschecking the reference lists of included CPGs. After the initial screening of the titles, 289 full texts were reviewed, out of which 264 were excluded (see Additional file 3). The most common reason for exclusion was that no QIs were reported. The remaining 25 international CPGs [23, 24, 28-50], originating from seven CPG providers, met the criteria for inclusion. The screening process is summarized in a flow chart (Fig. 1).

The 25 included international CPGs matched the topics of 18 of the 35 German S3-CPGs [16, 51-67]. Eight and three of the German S3-CPGs were developed by the GGPO and NDMG, respectively. Seven German S3-CPGs originated from other German medical 


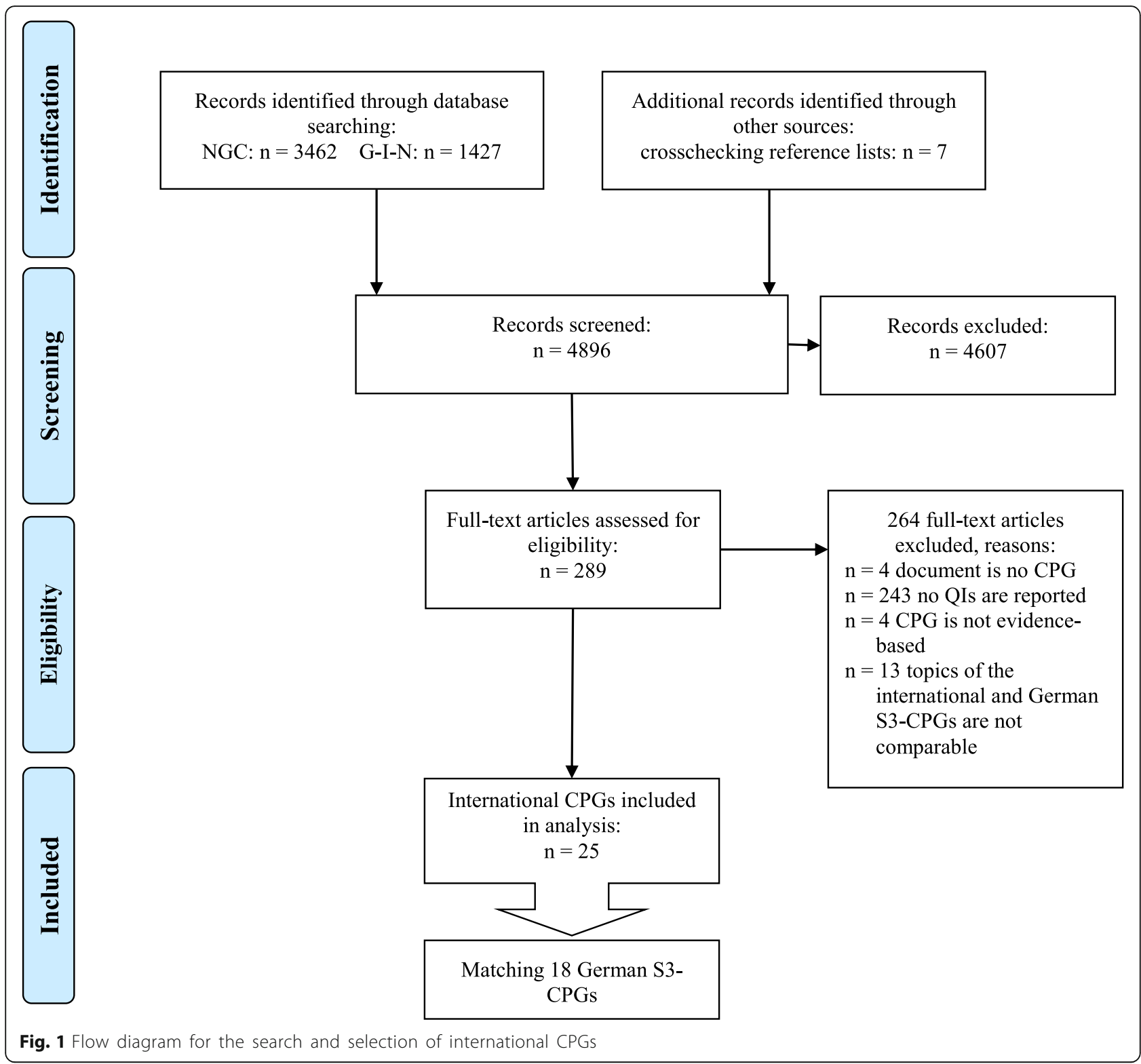

societies. We excluded those 17 German S3-CPGs from the analysis for which we found no eligible international CPG with matching topics. This resulted in 30 CPG pairs for the comparison of Qis, as some of the international CPGs matched the topic of more than one German S3-CPG. Table 1 gives an overview of the CPG pairs analysed.

Our assessment of methodological quality of the included CPGs gave a mean standardized score of $69 \%$ (standard deviation 7.8) for the domain "Methodological Rigor of Development" for the German S3-CPGs and $62 \%$ (standard deviation 12.7) for the international CPGs. For the individually rated items and resulting scores for each CPG, see Additional file 4.

\section{Characteristics of guideline-based QIs}

Overall, the German S3-CPGs and international CPGs contained 152 and 166 QIs on related topics, respectively. The median number of QIs per CPG was 8 (range 0-37) in the German S3-CPGs and 4.5 (range 1-15) in the international CPGs. With regard to the 30 CPG pairs, we compared 212 QIs from German S3-CPGs to 166 QIs from international CPGs (some of the QIs from German S3CPGs were counted more than once as we found more than one international CPG related to some German S3CPGs). The QIs in 85\% of German S3-CPGs (129 of 152) and $84 \%$ of international CPGs (139 of 166) were presented as ratios or proportions (defining numerator and denominator or quoting percentages). 
Table 1 CPG pairs identified

\begin{tabular}{|c|c|c|c|c|}
\hline CPG pair no. & Topic & German S3-CPG (abbreviation) & CPG pair $(n)$ & International CPG (abbreviation) \\
\hline 1 & Breast cancer & 032/045OL 2012 [61] & 1 & SIGN breast 2013 [42] \\
\hline 2 & Ovarian cancer & 032/035OL 2013 [16] & 1 & SIGN ovar 2013 [44] \\
\hline 3 & Prostate cancer & 043/022OL 2014 [51] & 1 & CTFPHC prostate 2014 [49] \\
\hline 4 & Colorectal cancer & 021/007OL 2013 [58] & 2 & CTFPHC colorectal 2016 [48] \\
\hline 5 & & & & SIGN colorectal 2016 [33] \\
\hline 6 & Oesophagus cancer & 021/023OL 2014 [64] & 1 & KCE gastrointest 2012 [28] \\
\hline 7 & Gastric cancer & 032/009OL 2012 [60] & 1 & KCE gastrointest 2012 [28] \\
\hline 8 & Palliative medicine & 128/001OL 2015 [65] & 1 & ICSI palliative 2013 [45] \\
\hline 9 & Melanoma & 032/024OL 2016 [62] & 1 & SIGN melanoma 2017 [43] \\
\hline 10 & Low-back pain & nvl/007 2015 [52] & 1 & ICSI backpain 2012 [39] \\
\hline 11 & Kidney disease in diabetes & nvl/001d 2015 [63] & 3 & NICE diabtypel 2015 [23] \\
\hline 12 & & & & NICE diabtypell 2016 [24] \\
\hline 13 & & & & SNS diabtypel 2012 [35] \\
\hline 14 & Diabetes training & nvl/001f 2012 [53] & 4 & NICE diabtypel 2015 [23] \\
\hline 15 & & & & NICE diabtypell 2016 [24] \\
\hline 16 & & & & SNS diabtypel 2012 [35] \\
\hline 17 & & & & ICSI diabtypell 2014 [36] \\
\hline 18 & Obesity & 050/001 2014*) [54] & 4 & CTFPHC obesity 2015 [50] \\
\hline 19 & & & & NICE obesity 2014 [29] \\
\hline 20 & & & & NICE weight 2014 [30] \\
\hline 21 & & & & ICSI obesity 2013 [31] \\
\hline 22 & Diabetes and pregnancy & $057-0232014^{*)}[56]$ & 2 & NICE diabpreg 2015 [34] \\
\hline 23 & & & & SNS diabtypel 2012 [35] \\
\hline 24 & Bipolar disorder & $\left.038-0192012^{*}\right)[55]$ & 1 & NICE bipolar 2016 [32] \\
\hline 25 & Hysterectomy for benign diseases & $\left.015-0702014^{*}\right)[57]$ & 1 & NICE menstrual bleeding 2016 [38] \\
\hline 26 & Long-term opioid-use in non-cancer pain & $145-0032014^{*)}[59]$ & 2 & ICSI pain 2016 [40] \\
\hline 27 & & & & SIGN pain 2013 [41] \\
\hline 28 & Venous thromboembolism & $003-0012015^{*}[66]$ & 2 & SIGN VTEPrev 2014 [47] \\
\hline 29 & & & & CCHMC VTE 2014 [46] \\
\hline 30 & Perioperative hypothermia & $001-0182013^{*)}[67]$ & 1 & ICSI hypo 2014 [37] \\
\hline
\end{tabular}

CCHMC Cincinnati Children's Hospital Medical Center, CTFPHC Canadian Task Force on Preventive Health Care, ICSI Institute for Clinical Systems Improvement, KCE Belgian Healthcare Knowledge Centre, NICE National Institute for Health and Care Excellence, SIGN Scottish Intercollegiate Guidelines Network, SNS (Spanish) National Health System, OL CPG of the German Guideline Program in Oncology, nvI CPG of the National Program for Disease Management Guidelines ${ }^{*}$ CPG of the scientific medical societies

In 17\% (3 of 18) of German S3-CPGs and 28\% (7 of 25) of international CPGs, a categorization of QIs into structure, process, or outcome indicators was made by the CPG authors themselves. According to our own assignment, we found mainly process indicators: 123 of 152 $(81 \%)$ in the German S3-CPGs and 133 of $166(80 \%)$ in the international CPGs. However, for 12 of 64 (19\%) QIs, we disagreed with the categorisation made by the authors of the international CPGs and therefore changed the category. For all nine QIs that were categorised by the authors of the German S3-CPGs, we agreed with the assignment.

The intended purpose of the QIs was reported in 13 of 18 (72\%) German S3-CPGs and in 21 of $25(84 \%)$ international CPGs. The rationale for the QIs was stated in only one of 18 (6\%) German S3-CPGs and in one of 25 (4\%) international CPGs.

An explicit link to one or more guideline recommendations was found for 136 of 152 (89\%) and 82 of 166 (49\%) QIs from 15 German S3 and 12 international CPGs, respectively.

Among these, 77\% (104 of 136) of QIs from German S3-CPGs and 93\% (76 of 82) from international CPGs were based on strong recommendations. Of these strong recommendations, $43 \%$ (45 of 104) in the German S3CPGs were consensus-based. This means they were based on the expert opinion of the CPG group, given 
that none or insufficient evidence exists for generating an evidence-based recommendation (in some CPGs those recommendations referred also to as "good clinical practice"). No recommendation in international CPGs was explicitly stated to be consensus-based, but all were evidence-based. However, the quality of the underlying evidence in five international CPGs from $\mathrm{KCE}$ and ICSI is mostly designated as "low" or "moderate". The underlying evidence of the strong recommendations in the seven included CPGs by NICE was mostly not clearly stated. For one of $152(0.7 \%)$ QIs in the German S3-CPGs and 23 of 166 (14\%) in the international CPGs, we found an implicit connection, as we identified one or more corresponding recommendation(s) in the particular CPG.

Quality objectives were stated for 39 of 152 (26\%) QIs in the German S3-CPGs and for 39 of 166 (23\%) QIs in the international CPGs. Properties were not reported for any QI measurement.

An overview of the QIs is presented in Fig. 2. Table 2 differentiates between responsible organisations within the German S3-CPGs.

\section{Comparison of Qls}

Twelve of the 30 CPG pairs comprised 27 QI pairs that were "not different or slightly different". This corresponds to $13 \%$ (27 of 212) of the QIs in German S3CPGs and 16\% (27 of 166) in international CPGs. Only two QI pairs were judged to be "different/inconsistent". For the majority of Qis, no direct comparison could be made, i.e. those QIs were found only in either the international or the German S3-CPGs (Table 3). Examples for all categories are presented in Table 4. All extracted QIs and corresponding recommendations can be found in Additional file 5 (QIs and recommendations out of German S3-CPGs were extracted only in German). Furthermore, a detailed comparison of all QIs on related topics is shown in Additional file 6 (the number of the QIs correspond to those stated in Additional file 5).

\section{Methods for the development of QIs}

Information on how QIs were developed was provided in 12 of 18 (67\%) German S3-CPGs and eight of 25 (32\%) international CPGs. Nine of the German S3-CPGs and one of the international CPGs [28] searched for and

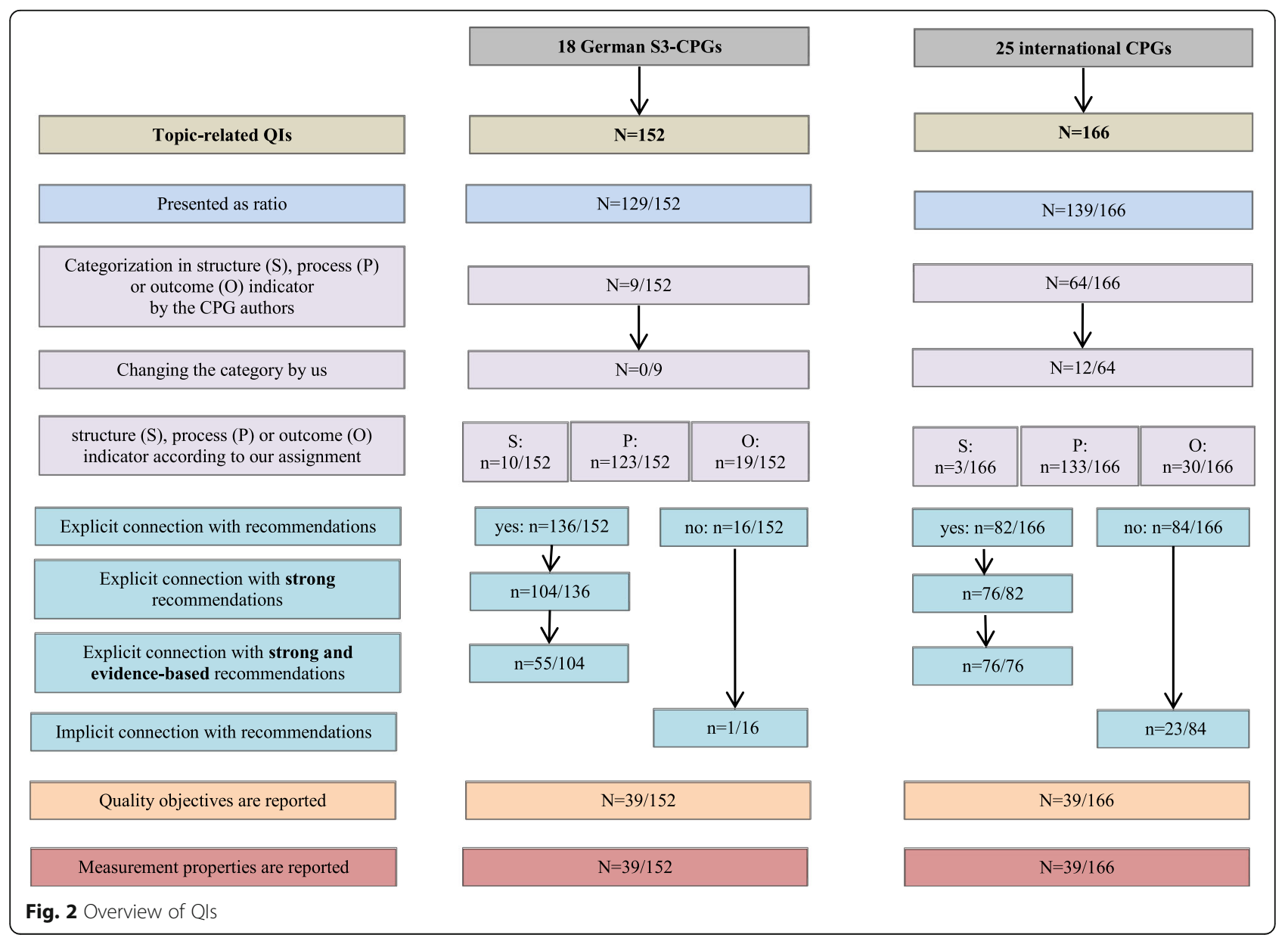


Table 2 Information on Qls with differentiation among German S3-CPGs

\begin{tabular}{|c|c|c|c|c|c|}
\hline & \multicolumn{3}{|l|}{ German S3-CPG } & \multirow{2}{*}{$\begin{array}{l}\text { German S3-CPG } \\
(n=18)\end{array}$} & \multirow{2}{*}{$\begin{array}{l}\text { International } \\
\text { CPG }(n=25)\end{array}$} \\
\hline & $\begin{array}{l}\text { Scientific medical } \\
\text { societies }(n=7)\end{array}$ & $\operatorname{NDMG}(n=3)$ & $\mathrm{GGPO}(n=8)$ & & \\
\hline \multicolumn{6}{|l|}{$\begin{array}{l}\text { Categorization of } \mathrm{Ql} \text { into structure }(\mathrm{S}) \text {, process }(\mathrm{P}) \text {, } \\
\text { or outcome }(\mathrm{O}) \text { indicator (according to own assignment) }\end{array}$} \\
\hline $\mathrm{S}(n) / \mathrm{P}(n) / \mathrm{O}(n)$ & $10 / 48 / 5$ & $0 / 14 / 0$ & $0 / 61 / 14$ & 10/123/19 & $3 / 133 / 30$ \\
\hline \multicolumn{6}{|l|}{ Intended purpose of QI is reported } \\
\hline Yes $(n)$ & 3 & 2 & 8 & 13 & 21 \\
\hline No $(n)$ & 4 & 1 & 0 & 5 & 4 \\
\hline \multicolumn{6}{|l|}{ Rationale for Ql is reported } \\
\hline Yes $(n)$ & 1 & 0 & 0 & 1 & 1 \\
\hline No $(n)$ & 6 & 3 & 8 & 17 & 24 \\
\hline \multicolumn{6}{|l|}{ QI is presented as ratio/proportion } \\
\hline$n / N$ & $40 / 63$ & $14 / 14$ & $75 / 75$ & 129/152 (85\%) & 139/166 (84\%) \\
\hline \multicolumn{6}{|l|}{ Ql is based explicitly on one or more recommendations } \\
\hline $\begin{array}{l}n / \mathrm{N} \\
\text { of that at least one strong recommendation/statement }\end{array}$ & $\begin{array}{l}53 / 63 \\
45 \%\end{array}$ & $\begin{array}{l}14 / 14 \\
100 \%\end{array}$ & $\begin{array}{l}69 / 75 \\
97 \%\end{array}$ & $\begin{array}{l}136 / 152(89 \%) \\
77 \%\end{array}$ & $\begin{array}{l}82 / 166(49 \%) \\
93 \%\end{array}$ \\
\hline \multicolumn{6}{|l|}{ Measurement properties are reported } \\
\hline$n / N$ & $0 / 63$ & $0 / 14$ & $0 / 75$ & $0 / 152$ & $0 / 166$ \\
\hline \multicolumn{6}{|l|}{ Quality objectives are reported } \\
\hline$n / N$ & $7 / 63$ & $7 / 14$ & $25 / 75$ & $39 / 152(26 \%)$ & $39 / 166(23 \%)$ \\
\hline
\end{tabular}

reported external data sources for QIs already in existence. Three international CPGs [37, 43, 44] referred to QIs that were developed by an institution that was not involved in the development of the particular CPG, such as the Scottish Cancer Taskforce. The application of formal methods for adopting existing QIs is reported in 12 of 18 (67\%) German S3-CPGs and in one of $25(4 \%)$ international CPGs. The use of formal criteria or tools to assess QIs is reported in 12 of 18 (67\%) German S3CPGs and in eight of 25 (32\%) international CPGs.

Regarding the underlying evidence for QIs in the German S3-CPGs of NDMG and GGPO, it is stated that QIs should be derived from strong recommendations. This methodological approach was implemented in 11 of the 18 (61\%) German S3-CPGs. None of the CPGs of the scientific medical societies gave information on underlying evidence. Among the international CPGs, eight of the 25 (32\%) CPGs originating from KCE and NICE provided information on which recommendation or grade of recommendation should be considered. For the KCE-CPG, it was explicitly stated that only strong recommendations were considered for the derivation of QIs. The NICE-
CPGs required proposed QIs to be linked by evidence to improved outcomes. For the remaining 17 international CPGs, no information was given.

None of the QIs from German S3-CPGs were reported to be piloted or evaluated, whereas eight international CPGs included a report on pilot testing during the development of QIs. Those international CPGs originated from only two CPG providers (KCE and NICE).

An overview on methodological aspects is presented in Table 5.

\section{Information on the composition of the QI development group}

Information on the composition of the QI development group was given in 14 of 18 (78\%) German S3-CPGs and in 12 of 25 (48\%) international CPGs. In the international CPGs, this information originated from three CPG providers (KCE, NICE, and SIGN). In four German S3-CPGs and 13 international CPGs, no information on the QI development group was given.

Clinicians, methodologists, and representatives of cancer registries were involved in the development of QIs of

Table 3 Comparison of Qls

\begin{tabular}{|c|c|c|c|c|}
\hline \multirow{2}{*}{$\begin{array}{l}30 \mathrm{CPG} \\
\text { pairs }\end{array}$} & \multicolumn{2}{|l|}{ QI match (QI pair) } & \multicolumn{2}{|l|}{ No match } \\
\hline & QI "not different or slightly different" & QI "different/inconsistent" & QI only in international CPG & QI only in German S3-CPG \\
\hline & $n=27$ & $n=2$ & $n=137$ & $n=183$ \\
\hline
\end{tabular}




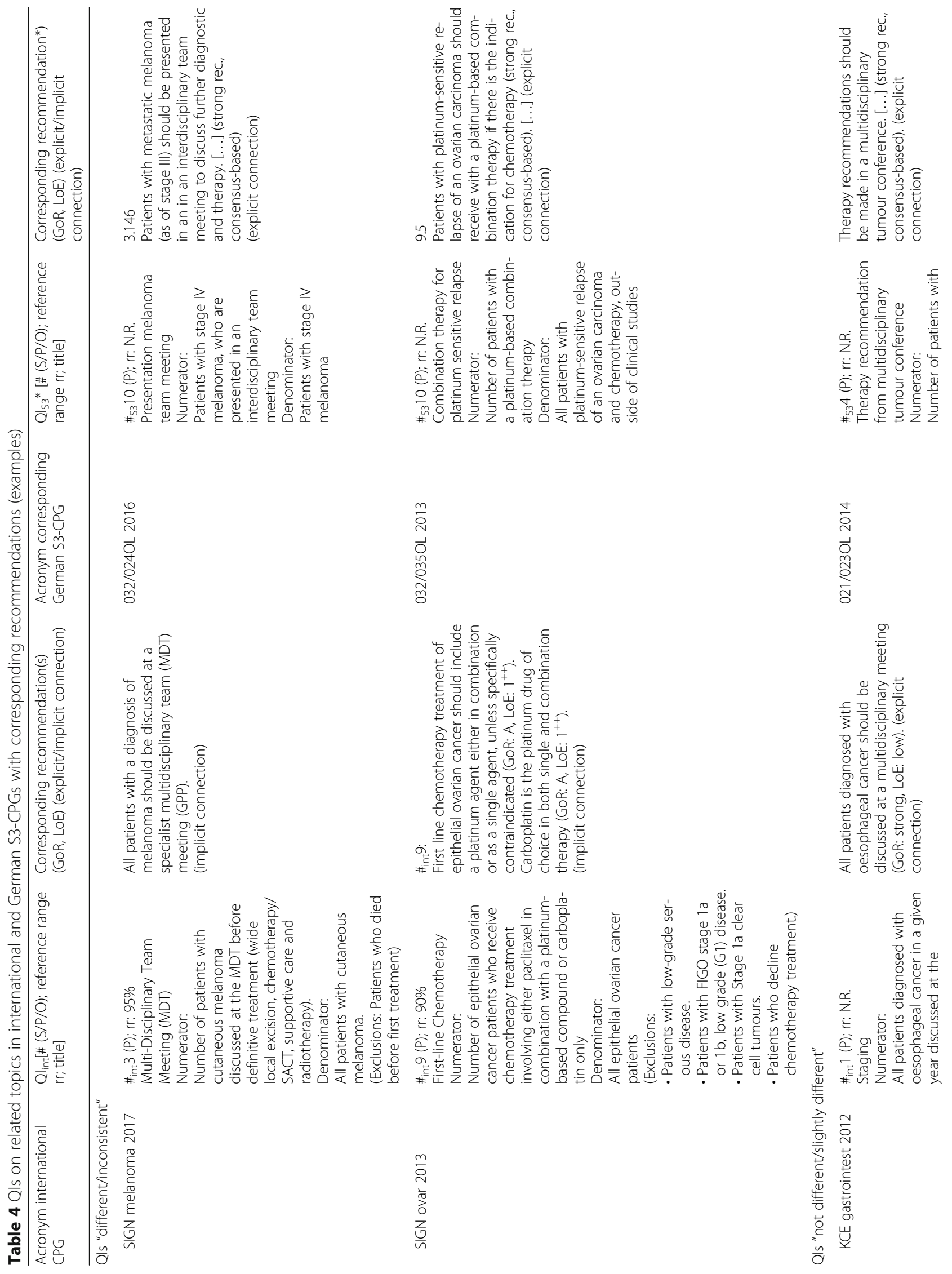




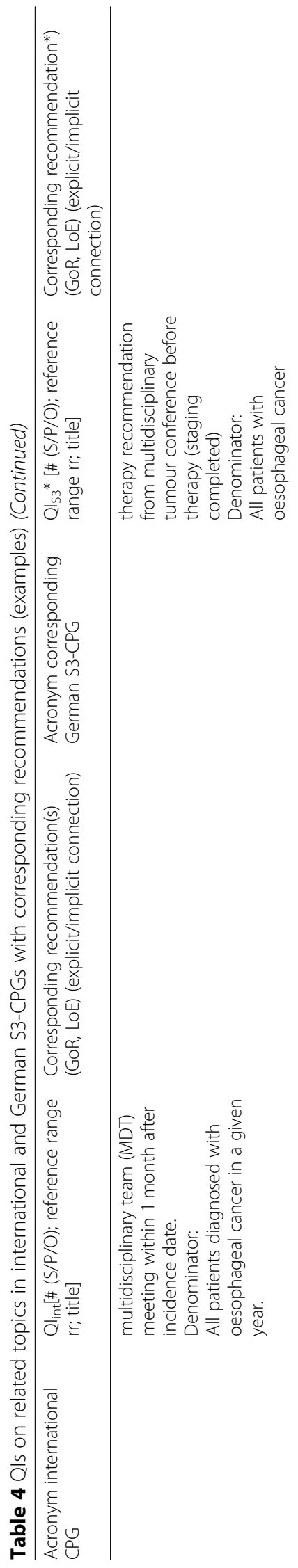

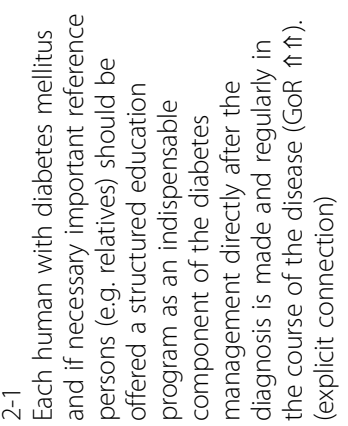

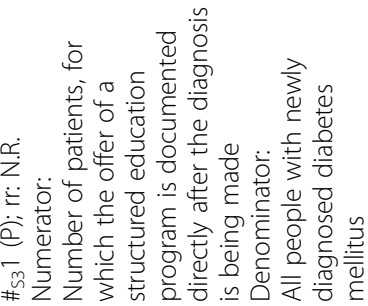

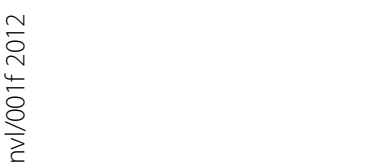

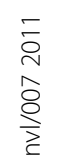

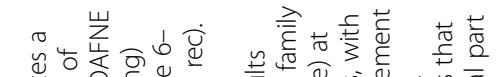

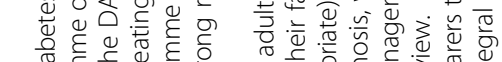

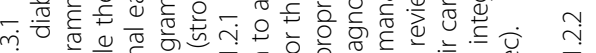
m-

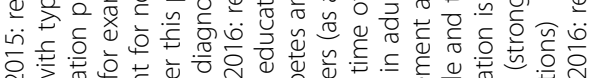

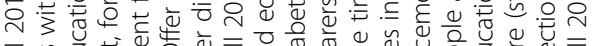

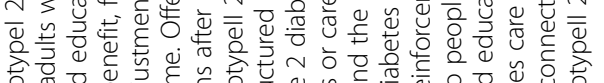

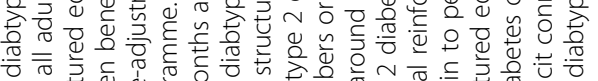

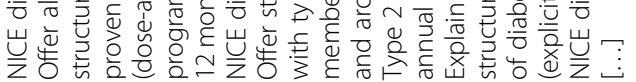
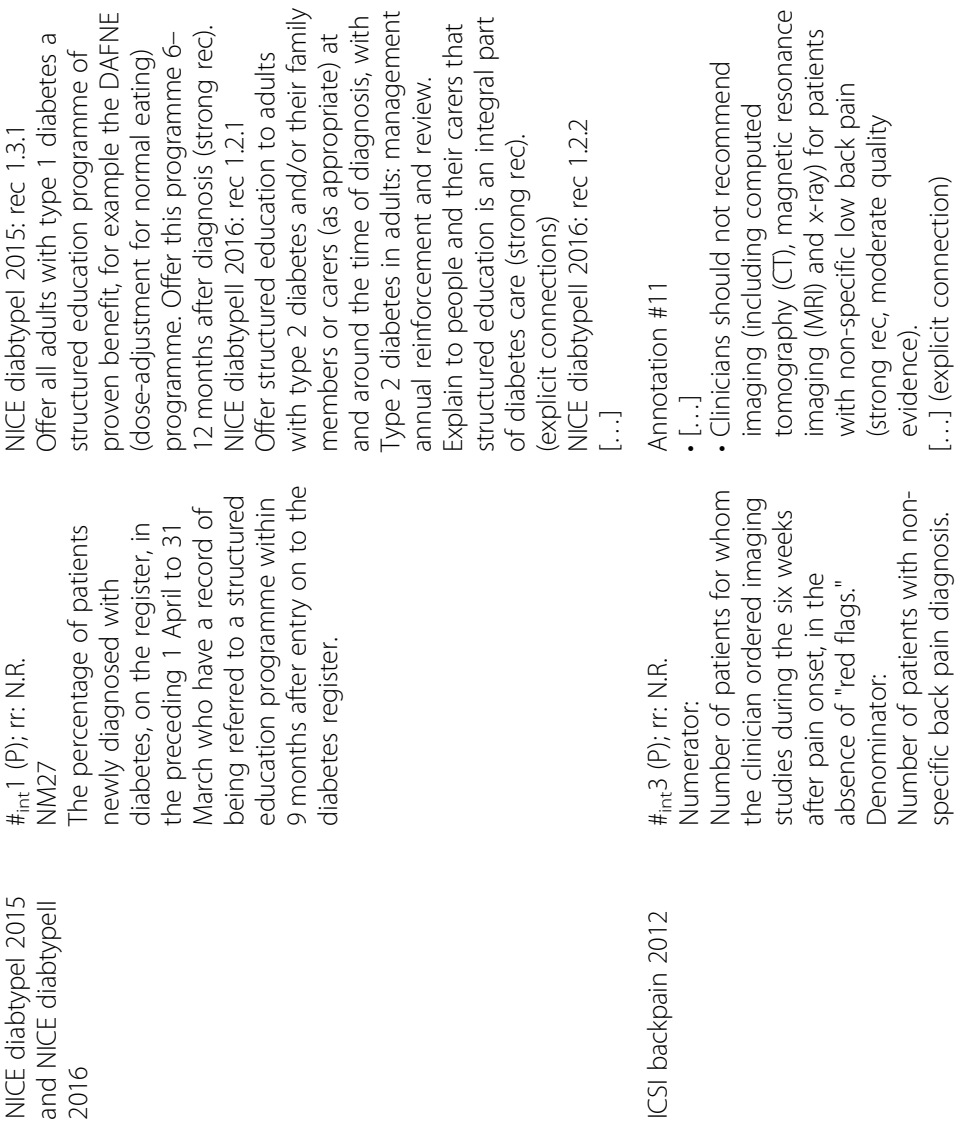

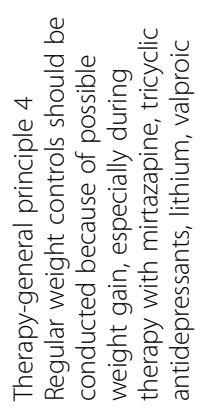

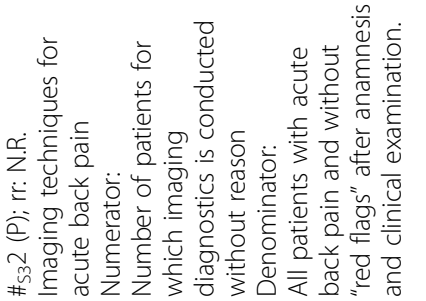

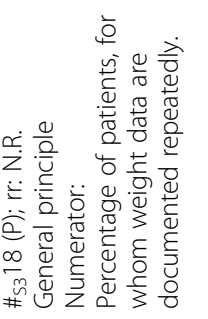

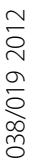

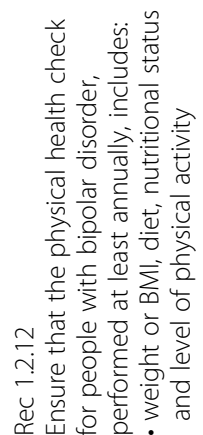

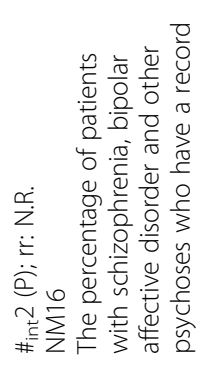




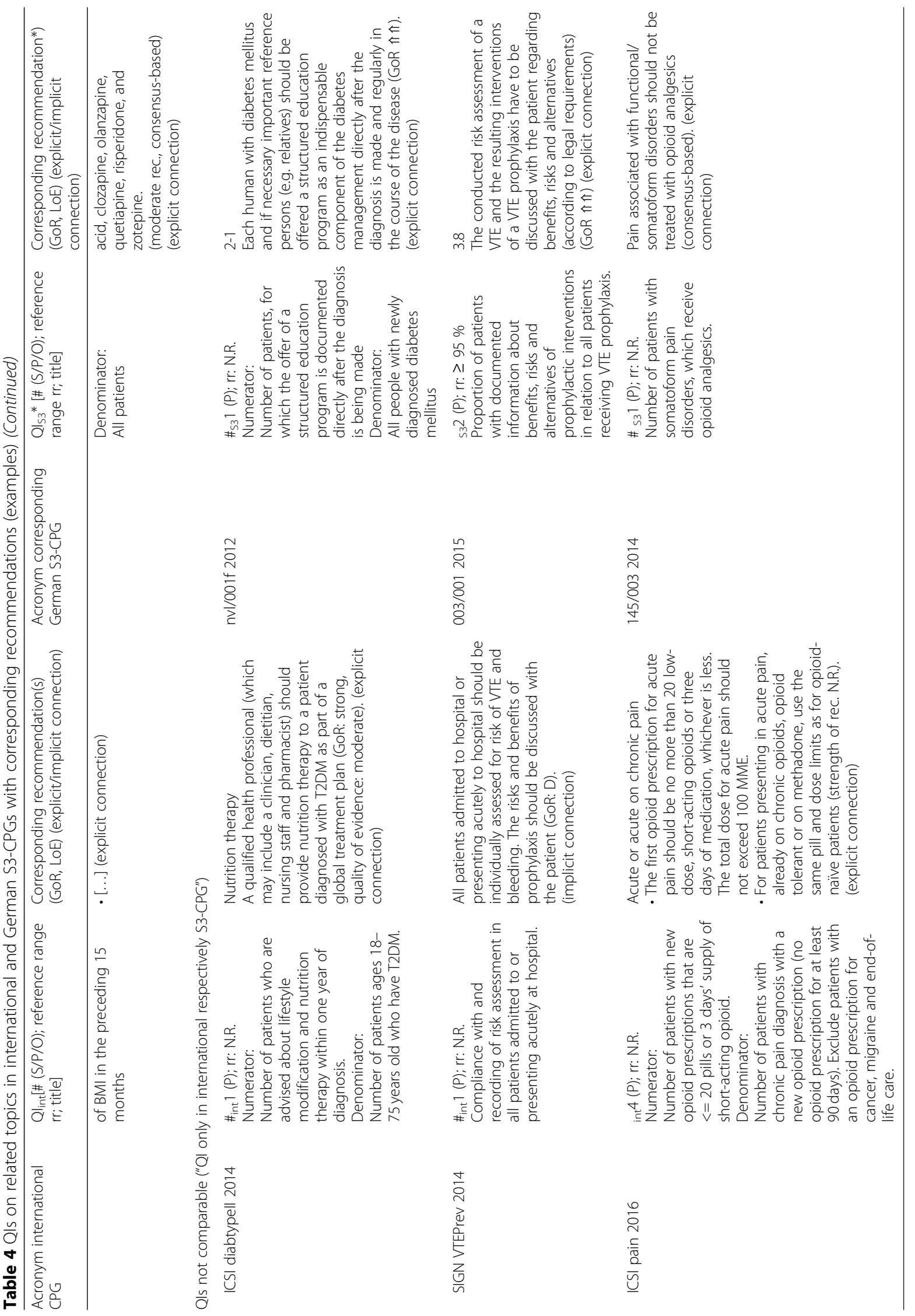


Table 5 Information on methodological aspects for development of guideline-based Qls

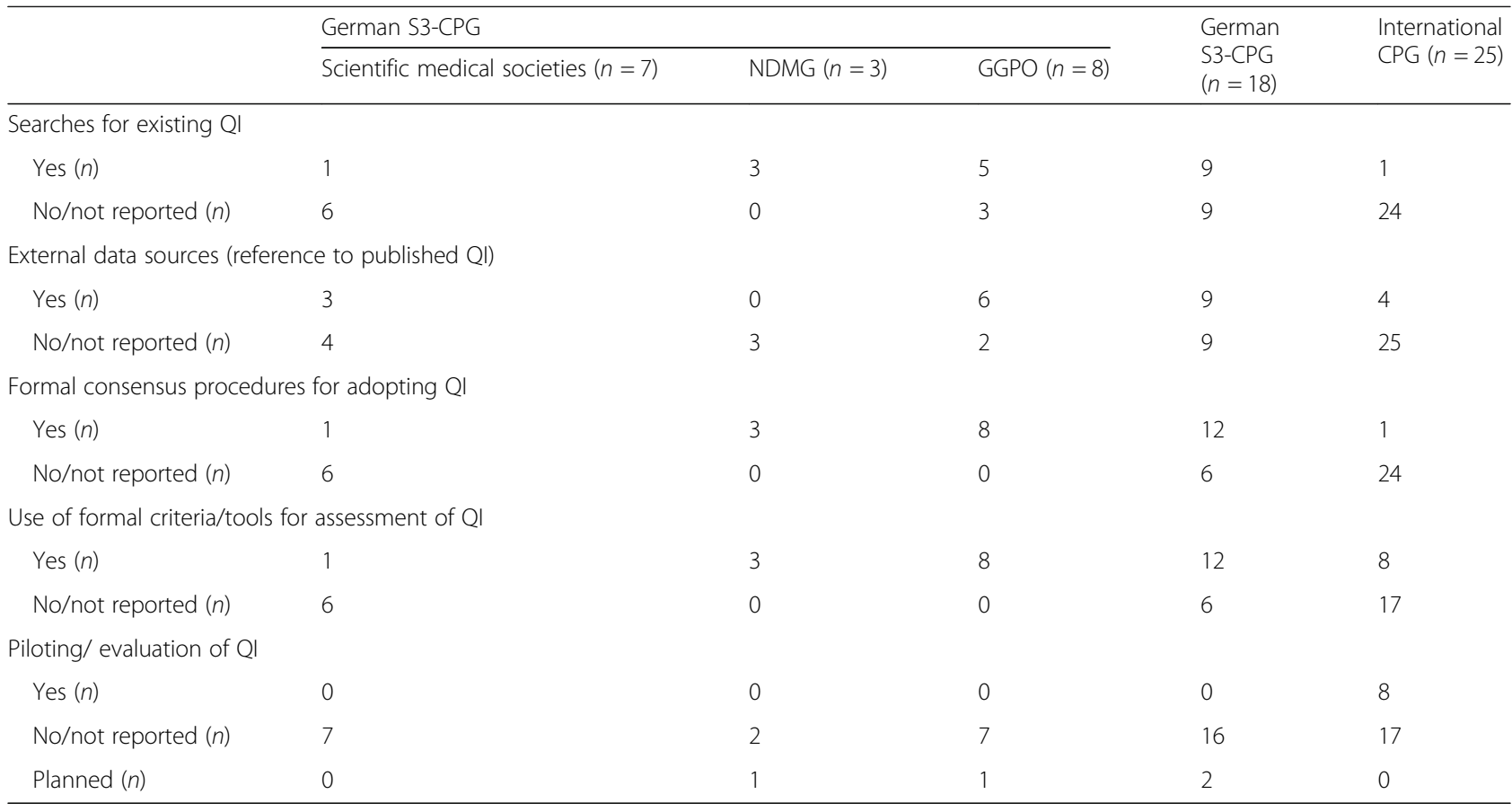

the KCE-CPG. According to the process guide of NICE, the QI development groups were multidisciplinary (e.g. clinicians, methodologists, public health and social care practitioners, patient representatives). However, there was no information on the actual composition of QI development groups for each individual included NICECPG. In one SIGN-CPG, it is stated that the QIs were defined by the CPG group.

Among the German S3-CPGs, all CPGs of the NDMG and GGPO and three CPGs developed by scientific medical societies gave information on the QI development group. For seven of the included German S3-CPGs, the QI development group comprised clinicians of different medical specialties, methodologists, and patient representatives, and in another seven German S3-CPGs, a participation of patient representatives was not reported.

An overview on the information on QIs, methods of development, and composition of QI development groups is given in an additional file for each included CPG (Additional file 7).

\section{Discussion}

Our analysis found that the majority of QIs in different CPGs on the same clinical topic was not comparable, but that they vary greatly in content and definitions. This result confirms our hypothesis that in many cases, QIs from German S3-CPGs do not correspond with QIs of international CPGs on related topics. However, only two QI pairs were rated as substantively "different/inconsistent". Although we suggested a hypothesis, we decided not to perform statistical testing due to the heterogeneous nature of the CPGs. They varied greatly for example in time period of literature searches, publication dates, developing organisation, and health care context as well as in the scope.

Detailed information on the methodological approach to generating QIs was lacking. Only two CPG providers of included international CPGs (NICE and KCE) reported information on the methods used to develop QIs. However, information was missing in these cases as well, such as reporting of the selection and extraction of CPG recommendations and their translation into QIs. Among the German S3-CPGs, all CPGs of the NDMG and the GGPO provided information on methods, whereas almost none of the CPGs of the medical societies contained methodological information. The quality appraisal score for the domain "Methodological Rigor of Development" ranged from 50 to $83 \%$ and from 48 to $83 \%$ in the German S3CPGs and international CPGs, respectively. High scores were not inevitably related to better description of the methods of developing the QIs or better reporting of QIs. Although it is assumed that the degree of credibility of QIs is associated with the methodological quality of CPGs, the evidence for this is lacking so far.

\section{Reasons for differences in QIs}

Various reasons are conceivable that would explain that QIs of different CPGs on the same clinical topic often did not cover the same quality aspect of care. One factor could be the different methodological approaches, e.g. to 
defining selection criteria for recommendations, to appraising the relevance of a QI for health care improvement, or to assessing feasibility of measurement. However, because it was rarely reported how QIs were generated (especially in the included international CPGs), we were unable to analyse this point in further detail. Therefore, for a better understanding of how guideline-based QIs are generated, a better reporting of the underlying processes is necessary. A proposal for reporting standards for guidelinebased performance measures has been developed by a working group of G-I-N [17].

Furthermore, although we compared only QIs on clinical topics that were addressed in both CPGs of a CPG pair, several recommendations of the German S3-CPGs and the related international CPGs varied to some extent in content and definitions. Most of the recommendations reported in international and German S3-CPGs were not inconsistent but had a different focus or depth of detail. For example, the German S3-CPG "Type 2 diabetes training" recommended to offer a structured education program, whereas the international CPG conducted by ICSI on "Diagnosis and Management of Type 2 Diabetes Mellitus in Adults" comprised a specific recommendation of nutrition therapy. Nutrition therapy was also considered in the particular German S3CPG within the explanatory text. However, no specific recommendation on nutrition was made. Further, there were other cases where both CPGs of a CPG pair comprised comparable recommendations, but only in one of the CPGs, a QI was derived from the recommendation(s).

Also, different definitions of QIs may result from an inconsistent composition of the QI development groups with methodologists, relevant health care professionals, stakeholders, and patients. A study about the consistency of QI selection for cardiovascular risk management across different consensus methods and panels found, in part, considerable variation, but could not explain the underlying factors [68]. Further reasons may relate to contextual differences between countries and different health care problems. Regarding guideline-based QIs, another factor could be the up-to-dateness of the CPGs. Many CPGs become out-of-date after about five years [69]. However, in fast-evolving medical fields, recommendations could become out-of-date even earlier.

The analyses of the two inconsistent QI pairs found that the underlying recommendations are also inconsistent, even though the link between QI and recommendation in the international CPG is only implicit. For example, the SIGN-CPG on ovarian cancer recommends that first-line chemotherapy should include a platinum agent either in combination or as a single agent [44], whereas the German S3-CPG recommends solely platinum-based combination therapy [16]. For inconsistent recommendations, various reasons are conceivable likewise, such as differences in the underlying evidence that was used, in the assessment of the evidence, in the composition of the CPG development group, and in value judgements as well the health care context.

\section{Studies comparing QIs from different countries}

Studies on the transferability of non-guideline-based QIs between the USA and the UK and between the USA and the Netherlands found that about $56 \%$ and $67 \%$, respectively, were "exactly or nearly equivalent" or "(nearly) identical" [70, 71]. According to the authors, the main reasons for differences seemed to be related to differences in clinical practice or variation in professional culture and expert opinion. The consistency between QIs in our analysis is considerably smaller: only $13 \%$ of the QIs in the German S3-CPGs had international equivalents. This discrepancy may be explained by the fact that our analysis focused solely on guideline-based QIs, whereas the QIs in the other studies were derived from a broader literature basis. Furthermore, disparities may be explained by different categorisation of QIs as "nearly equivalent/identical" and "slightly different". However, this aspect is difficult to assess as definitions for "nearly equivalent/identical" are missing in the studies. In a recent study, Petzold et al. (2018) compared QIs from German S3-CPG with quality measures in NICE quality standards [72]. NICE quality standards consist of statements designed for quality improvements within a particular area of health, each statement being related to quality measures which support their implementation [73]. They are based on NICE guidelines and other NICE-accredited guidance [73]. NICE indicators also measure outcomes considered to reflect the quality of care or processes [73]. In contrast to the quality measures in NICE quality standards, the latter are generally linked directly to specific NICE CPG recommendations. Petzold et al. found that only 34 of 128 (27\%) German QIs and 34 of 468 (7\%) NICE quality measures they analysed related to the same medical problem [72]. As in our analysis, the consistency between QIs is considerably smaller than in the studies on the transferability of nonguideline-based QIs from different countries. However, the results in the study of Petzold et al. correspond only modestly with the results of our analysis, even if we would separate the NICE-CPGs in our analysis. This could be explained by the fact that we only considered QIs that are directly linked with a CPG as reported, for example, in the "NICE indicator menu" [22]. Petzold et al. exclusively considered quality measures in NICE quality standards that are relevant to NICE-CPGs. We did not consider those because the connection between quality measure and CPG is only indirect. As a result, the two analyses included different German S3-CPGs and NICE-CPGs and, accordingly, different QIs. 


\section{QIs and the underlying evidence}

Our analysis found that over $40 \%$ of the strong recommendations in the German S3-CPGs are based exclusively on the expert opinion of the CPG group. Furthermore, the quality of the underlying evidence of many strong recommendations in the international CPGs was designated as "low" or "moderate". This appears to contradict the methodological requirement that QIs should be based on scientific evidence, where possible $[7,8]$. However, it might seem reasonable to derive QIs from expert opinion in cases where none or limited evidence exists and a great potential for quality improvement is seen nevertheless by the CPG group. In cases of strong evidence-based recommendations with low or moderate quality of the evidence, it should be noted that various criteria other than the underlying evidence influence the decision about the grade of recommendation, such as clinical relevance, practical experience, riskbenefit ratio, and applicability to clinical practice. The Grading of Recommendations Assessment, Development, and Evaluation (GRADE) system of rating the quality of evidence and grading the strength of recommendations in CPGs, for example, offers a transparent and structured process for developing recommendations $[74,75]$. Thus, the application of GRADE or related systems is seen to increase.

\section{QI development group}

Especially in the international CPGs, information on the composition and responsibilities of the QI development group is lacking. Further understanding of the interaction between the QI and CPG development groups are needed, if they work independently. In this context, cooperation and mutual feedback between these stakeholders are reasonable. For example, the QI development group might need further background information regarding recommendations, or the results of the QI development could lead to a revision of recommendations.

\section{Piloting and evaluation of QI}

None of the QIs from German S3-CPGs were piloted or evaluated. However, this step ought to be seen as an essential element in the process of developing QIs [73, 76]. To assess the usefulness of potential QIs, information on criteria including technical feasibility, reliability, and validity is necessary. Such data can be generated only by testing the QIs in routine care [77]. Accordingly, several literature and protocols regarding the piloting and evaluation of QIs in general (not only guideline-based) are available [77-80].

\section{Strength and limitations of the review}

The strength of our analyses is the systematic methodological approach which followed a pre-defined protocol.
However, although we conducted systematic literature searches in the two main guideline databases, we may have missed CPGs not included in the databases. A further limitation of our analysis is that we probably missed information on methodological issues from further CPG providers, as we only included CPGs that matched substantively with a German S3-CPG. Furthermore, potential limitations arise from the fact that both the selection of CPGs and data extraction were performed by only one reviewer and checked by another. This pragmatic approach was chosen because of the large number of hits obtained by the diverse searches, as well as the low level of complexity regarding inclusion criteria in our study. Moreover, the data extraction is in agreement with a recent methodological guide on systematic reviews of CPGs [81].

We did not analyse the aspect of evidence underlying the QIs in the German S3- and international CPGs in depth, as we found various systems rating the quality of evidence and grading the strength of recommendations in the CPGs.

Finally, the interpretability of our results might be limited as we compared the QIs on clinical topics that were addressed in both CPGs of a CPG pair directly, rather than at the recommendation level. As noted above, although the CPG pairs addressed the same clinical topics, the recommendations varied to some extent and, in some cases, resulted in QIs that were not comparable. However, it should be noted that only about half of the QIs reported in international CPGs were based explicitly on guideline recommendations. The underlying approaches for generating such QIs were not reported in sufficient detail.

\section{Conclusion}

The majority of QIs in German and international CPGs were not comparable. Various reasons for this are conceivable, such as methodological issues or contextual differences between countries. However, no clear reason could be deduced from the available data. Detailed information on the methodological approaches of generating QIs is lacking. More transparent reporting of the underlying methods for generating guideline-based QIs is recommended.

\section{Additional files}

Additional file 1: German S3-CPGs which report Qls. (DOCX 27 kb)

Additional file 2: Search strategies. (DOCX $17 \mathrm{~kb}$ )

Additional file 3: Excluded articles. (DOCX $55 \mathrm{~kb}$ )

Additional file 4: Quality appraisal of included CPGs. (DOCX 35 kb)

Additional file 5: Topic-related Qls in international CPGs and German S3CPGs and if applicable corresponding recommendations. (DOCX 238 kb) 
Additional file 6: Comparison of topic-related Ols in international CPGs and German S3-CPGs. (DOCX 37 kb)

Additional file 7: Table a. Data-Extraction of German S3-CPGs - general and methodical aspects and Table $\mathbf{b}$. Data-Extraction of international CPGs - general and methodical aspects. (DOCX 60 kb)

\section{Abbreviations}

AWMF: German Association of the Scientific Medical societies: CPG: Clinical practice guideline; DELBI: German Instrument for Methodological Guideline Appraisal; GGPO: German Guideline Program in Oncology; G-I-N: Guidelines International Network; GoR: Grade of Recommendation; LoE: Level of Evidence; NDMG: National Program for Disease Management Guidelines; NGC: National Guideline Clearinghouse; NICE: National Institute for Health and Care Excellence; QI: Quality indicator

\section{Acknowledgements}

We thank Max Geraedts (University of Marburg, Germany) for reviewing the final draft of the review and Käthe Goossen (Witten/Herdecke University, Germany) for language editing of the final draft.

\section{Authors' contributions}

EN, JS, and MN contributed to the initial study idea. All authors contributed to the conception and design of the study. MBe and JB performed the literature search and screening, literature retrieval, data extraction and interpretation, and quality appraisal of CPGs. DP contributed to the data interpretation and supervised the process. MN contributed to literature screening. $\mathrm{MBr}$ and SD contributed to the quality appraisal of German S3CPGs and extraction of Qls from German S3-CPGs. MBe wrote the first draft of the manuscript. All authors have been involved in critically reviewing and revising the manuscript and approved the final manuscript.

\section{Funding}

German Research Association (DFG) (grant no. NE 385/23-1)

\section{Availability of data and materials}

All data generated or analysed during this study are included in this published article and its supplementary information files.

\section{Ethics approval and consent to participate}

Not applicable

\section{Consent for publication}

Not applicable

\section{Competing interests}

MN was involved in the development of several German S3-CPGs that are considered in this review. MBe was involved in the preparation of the evidence report for one CPG that is considered in this review. MBe, JB, JS, EN, and DP were involved in the development of several German S3-CPGs that are not considered in this review. SD and $\mathrm{MBr}$ declare no competing interests (financial and non-financial).

\section{Author details \\ ${ }^{1}$ Institute for Research in Operative Medicine (IFOM), Department Evidence-based health services research, Faculty of Health, Department of Medicine, Witten/Herdecke University, Ostmerheimer Str. 200, Building 38, 51109 Cologne, Germany. ${ }^{2}$ AWMF-Institute for Medical Knowledge Management c/o Philipps-University Marburg, Karl-von-Frisch-Straße 1, 35043 Marburg, Germany. ${ }^{3}$ Center for Evidence-based Healthcare, University Hospital and Medical Faculty Carl Gustav Carus, TU Dresden, Fetscherstraße 74, 01309 Dresden, Germany. ${ }^{4}$ Brandenburg Medical School - Theodor Fontane, Fehrbelliner Str.38, 16816 Neuruppin, Germany.}

Received: 31 January 2019 Accepted: 18 June 2019 Published online: 09 July 2019

\section{References}

1. Lawrence M, Frede O. Indicators of quality in health care. Eur J Gen Pract. 1997:3:103-8
2. The Joint Commission on Accreditation of Healthcare Organizations (JCAHO). Characteristics of clinical indicators. QRB Qual Rev Bull. 1989;15(11): 330-9.

3. K Edward and $\mathrm{H}$. Jermy, Health care quality indicators project conceptual framework paper. 2006: OECD Health Working Papers: 23, https://www. oecd.org/els/health-systems/36262363.pdf. Accessed 08 May 2017.

4. Reiter A, et al. QUALIFY--a tool for assessing quality indicators. Z Arztl Fortbild Qualitatssich. 2007;101(10):683-8.

5. National Quality Forum. Measure evaluation criteria and guidance for evaluating measures for endorsement. 2016. http://www.qualityforum.org/ Measuring_Performance/Endorsed_Performance_Measures_Maintenance. aspx. Accessed 08 May 2017.

6. Geraedts M, Selbmann HK, Ollenschlaeger G. Critical appraisal of clinical performance measures in Germany. Int J Qual Health Care. 2003;15(1):79-85.

7. Campbell SM, et al. Research methods used in developing and applying quality indicators in primary care. Qual Saf Health Care. 2002;11(4):358-64.

8. Mainz J. Defining and classifying clinical indicators for quality improvement Int J Qual Health Care. 2003;15(6):523-30.

9. Institute of Medicine (U.S.). Committee on Redesigning Health Insurance Performance Measures, P., and Performance Improvement Programs, Performance measurement: accelerating improvement (Pathways to Quality Health Care Series). 2006, The National Academies Press Washington, DC: http://www.nap.edu. Accessed 08 May 2017.

10. Kötter T, Blozik E, Scherer M. Methods for the guideline-based development of quality indicators--a systematic review. Implement Sci. 2012;7:21.

11. German Guideline Program in Oncology (German Cancer Society, German Cancer Aid, Association of the Scientific Medical Societies). Development of guideline-based quality indicators: methodology for the German Guideline Program in Oncology, version 2.0 2017. http://leitlinienprogramm-onkologie. de/methodik/information-zur-methodik/. Accessed 25 June 2019.

12. German Medical Association (GMA), National Association of Statutory Health Insurance Physicians (NASHIP), and Association of the Scientific Medical Societies (AWMF). National Programme for Disease Management Guidelines. Method Report. 4th. 2010. http://www.versorgungsleitlinien.de/methodik/ reports. Accessed 08 May 2017.

13. Nothacker MJ, Langer T, Weinbrenner S. Quality indicators for Nationa Disease Management Guidelines using the example of the National Disease Management Guideline for "Chronic Heart Failure". Z Evid Fortbild Qual Gesundhwes. 2011;105(1):27-37.

14. Arbeitsgemeinschaft der Wissenschaftlichen Medizinischen Fachgesellschaften (AWMF)- Ständige Kommission Leitlinien, AWMF-Regelwerk "Leitlinien". 2012. http://www.awmf.org/leitlinien/awmf-regelwerk.html. Accessed 08 May 2017.

15. Schmitt J, et al. Recommendations for quality indicators in German S3 guidelines: a critical appraisal. Gesundheitswesen. 2014;76(12):819-26.

16. Leitlinienprogramm Onkologie (Deutsche Krebsgesellschaft, Deutsche Krebshilfe, AWMF). S3-Leitlinie Diagnostik, Therapie und Nachsorge maligner Ovarialtumoren, Langversion 1.0 2013 AWMF Registrierungsnummer: 032035OL. http://leitlinienprogramm-onkologie.de/Leitlinien.7.0.html.

17. Nothacker $\mathrm{M}$, et al. Reporting standards for guideline-based performance measures. Implement Sci. 2016;11:6.

18. Blozik $E$, et al. Simultaneous development of guidelines and quality indicators -- how do guideline groups act? A worldwide survey. Int J Health Care Qual Assur. 2012:25(8):712-29.

19. Moher D, et al. Preferred reporting items for systematic reviews and metaanalyses: the PRISMA statement. PLoS Med. 2009;6(7):e1000097.

20. Becker $M$, et al. Guideline-based quality indicators-a systematic comparison of German and international clinical practice guidelines: protocol for a systematic review. Syst Rev. 2018;7(1):5

21. World Health Organization. List of Member States by WHO Region and Mortality Stratum. http://www.who.int/choice/demography/mortality_strata/ en/. Accessed 20 Oct 2017

22. National Institute for Health and Care Excellence (NICE). The NICE menu of general practice and clinical commissioning group indicators. 2018. https:// www.nice.org.uk/standards-and-indicators. Accessed 25 June 2019.

23. National Institute for Health and Clinical Excellence. Type 1 diabetes in adults: diagnosis and management. 2015. http://nice.org.uk/guidance/ng17. Accessed 07 July 2017

24. National Institute for Health and Clinical Excellence. Type 2 diabetes in adults: management. 2015. http://nice.org.uk/guidance/ng28. Accessed 21 Mar 2017.

25. Donabedian A. The role of outcomes in quality assessment and assurance. QRB Qual Rev Bull. 1992;18(11):356-60. 
26. Mokkink LB, et al. The COSMIN checklist for assessing the methodological quality of studies on measurement properties of health status measurement instruments: an international Delphi study. Qual Life Res. 2010;19(4):539-49.

27. German Association of the Scientific Medical societies (AWMF) and Agency for Quality in Medicine (ÄZQ). German Instrument for Methodological Guideline Appraisal 2008: http://www.delbi.de/. Accessed 08 May 2017.

28. Lerut T, Stordeur S, Verleye L, Vlayen J, Boterberg T, De Hertogh G, De Mey J, Deprez P, Flamen P, Pattyn P, Van Laethem J-L, Peeters M. Update van de praktijkrichtlijn voor slokdarm- en maagkanker. Good Clinical Practice (GCP). Brussel: Federaal Kenniscentrum voor de Gezondheidscentrum (KCE). KCE Report 179A. D/2012/10.273/32. 2012. https:/kce.fgov.be/en/update-of-the-nationalguideline-on-upper-gastrointestinal-cancer. Accessed 15 Sept 2017.

29. National Institute for Health and Clinical Excellence. Obesity: identification, assessment and management. 2014. http://nice.org.uk/guidance/cg189. Accessed 14 Mar 2017

30. National Institute for Health and Clinical Excellence. Weight management: lifestyle services for overweight or obese adults. 2014. http://nice.org.uk guidance/ph 53. Accessed 16 Mar 2017.

31. Fitch A, Everling L, Fox C, Goldberg J, Heim C, Johnson K, Kaufman T, Kennedy E, Kestenbaun C, Lano M, Leslie D, Newell T, O'Connor P, Slusarek B, Spaniol A, Stovitz S, Webb B. Institute for Clinical Systems Improvement. Prevention and Management of Obesity for Adults. Updated May 2013.

32. National Institute for Health and Clinical Excellence. Bipolar disorder: assessment and management. 2014. http://nice.org.uk/guidance/cg185. Accessed 03 Mar 2017

33. Scottish Intercollegiate Guidelines Network (SIGN). Diagnosis and management of colorectal cancer. Edinburgh: SIGN; 2011. (SIGN publication no. 126). [Revised August 2016]. http://www.sign.ac.uk. Accessed 26 Mar 2017.

34. National Institute for Health and Clinical Excellence. Diabetes in pregnancy: management from preconception to the postnatal period. 2015. http://nice. org.uk/guidance/ng3. Accessed 21 Mar 2017.

35. Working Group of the Clinical Practice Guideline on Diabetes mellitus type 1. Clinical Practice Guidelines for Diabetes mellitus type 1. Quality Plan for the National Health Service of the Ministry of Health and Social Policy. Agency for Health Technology Assessment from the Basque Country -Osteba, 2012. http://www.guiasalud.es/GPC/GPC_513_Diabetes_1_Osteba_ compl_en.pdf. Accessed 21 Mar 2017.

36. Redmon B, Caccamo D, Flavin P, Michels R, O'Connor P, Roberts J, Smith S, SperlHillen J. Institute for Clinical Systems Improvement. Diagnosis and management of type 2 diabetes mellitus in adults. Updated July 2014. https://mww.icsi.org/ guidelines_more/catalog_guidelines_and_more/catalog_guidelines/catalog_ endocrine_guidelines/diabetes/. Accessed 21 Mar 2017.

37. Card R, Sawyer M, Degnan B, Harder K, Kemper J, Marshall M, Matteson M, Roemer R, Schuller-Bebus G, Swanson C, Stultz J, Sypura W, Terrell C, Varela N. Institute for Clinical Systems Improvement. Perioperative protocol. Updated March 2014. https://www.icsi.org/guidelines_more/catalog_ guidelines_and_more/catalog_guidelines/catalog_patient_safetyreliability_ guidelines/perioperative/. Accessed 20 Mar 2017

38. National Institute for Health and Clinical Excellence. Heavy menstrual bleeding: assessment and management. Update August 2016. http://nice. org.uk/guidance/cg44. Accessed 30 Mar 2017.

39. Goertz M, Thorson D, Bonsell J, Bonte B, Campbell R, Haake B, Johnson K, Kramer C, Mueller B, Peterson S, Setterlund L, Timming R. Institute for Clinical Systems Improvement. Adult acute and subacute low back pain. Updated November 2012

40. Hooten M, Thorson D, Bianco J, Bonte B, Clavel Jr A, Hora J, Johnson C, Kirksson E, Noonan MP, Reznikoff C, Schweim K, Wainio J, Walker N. Institute for Clinical Systems Improvement. Pain: Assessment, non-opioid treatment approaches and opioid management. Updated September 2016. https://www. icsi.org/guidelines_more/catalog_guidelines_and_more/catalog_guidelines/ catalog_neurological_quidelines/pain/. Accessed 09 June 2017.

41. Scottish Intercollegiate Guidelines Network (SIGN). Management of chronic pain. Edinburgh: SIGN; 2013. (SIGN publication no. 136). http://www.sign.ac. uk. Accessed 03 Mar 2017

42. Scottish Intercollegiate Guidelines Network (SIGN). Treatment of primary breast cancer. Edinburgh: SIGN; 2013. (SIGN publication no. 134). http:// www.sign.ac.uk. Accessed 24 Mar 2017.

43. Scottish Intercollegiate Guidelines Network (SIGN). Cutaneous melanoma. Edinburgh: SIGN; 2017. (SIGN publication no. 146). http://www.sign.ac.uk. Accessed 21 Mar 2017.
44. Scottish Intercollegiate Guidelines Network (SIGN). Management of epithelial ovarian cancer. Edinburgh: SIGN; 2013. (SIGN publication no. 135). http://www.sign.ac.uk. Accessed 26 Mar 2017.

45. McCusker M, Ceronsky L, Crone C, Epstein H, Greene B, Halvorson J, Kephart K, Mallen E, Nosan B, Rohr M, Rosenberg E, Ruff R, Schlecht K, Setterlund L. Institute for Clinical Systems Improvement. Palliative care for adults. Updated November 2013.

46. Multidisciplinary VTE Prophylaxis BESt Team, Cincinnati Children's Hospital Medical Center: Best evidence statement venous thromboembolism (VTE) prophylaxis in children and adolescents. http://www.cincinnatichildrens.org/ service/j/anderson-center/evidence-based-care/bests/. BESt 181, pages 1-14, Date 2/18/14. Accessed 20 March 2017

47. Scottish Intercollegiate Guidelines Network (SIGN). Prevention and management of venous thromboembolism. Edinburgh: SIGN; 2010. (SIGN publication no. 122). [updated Oct 2014]. http://www.sign.ac.uk. Accessed 07 June 2017

48. Bacchus CM, et al. Recommendations on screening for colorectal cancer in primary care. CMAJ. 2016;188(5):340-8.

49. Bell N, et al. Recommendations on screening for prostate cancer with the prostate-specific antigen test. CMAJ. 2014;186(16):1225-34.

50. Brauer $\mathrm{P}$, et al. Recommendations for prevention of weight gain and use of behavioural and pharmacologic interventions to manage overweight and obesity in adults in primary care. CMAJ. 2015;187(3):184-95.

51. Leitlinienprogramm Onkologie (Deutsche Krebsgesellschaft, Deutsche Krebshilfe, AWMF). Interdisziplinäre Leitlinie der Qualität S3 zur Früherkennung, Diagnose und Therapie der verschiedenen Stadien des Prostatakarzinoms, Langversion 3.1 2014 AWMF Registernummer: 034/ 022OL. http://leitlinienprogramm-onkologie.de/Leitlinien.7.0.html. Accessed 19 June 2017.

52. Bundesärztekammer (BÄK), Kassenärztliche Bundesvereinigung (KBV), Arbeitsgemeinschaft der Wissenschaftlichen Medizinischen Fachgesellschaften (AWMF). Nationale VersorgungsLeitlinie Kreuzschmerz Langfassung, 1. Auflage. Version 5. 2010, zuletzt verändert: Oktober 2015. AWMF Registernummer: nvl/007. http://www.kreuzschmerz. versorgungsleitlinien.de. Accessed 19 June 2017.

53. Bundesärztekammer (BÄK), Kassenärztliche Bundesvereinigung (KBV), Arbeitsgemeinschaft der Wissenschaft-lichen Medizinischen Fachgesellschaften (AWMF). Nationale VersorgungsLeitlinie Diabetes Strukturierte Schulungsprogramme - Langfassung, 1. Auflage. Version 4. 2012. AWMF Registernummer: nvl-001f. http://www.dm-schulung. versorgungsleitlinien.de. Accessed 19 June 2017.

54. Deutsche Adipositas-Gesellschaft (DAG). Interdisziplinäre Leitlinie der Qualität S3 zur, "Prävention und Therapie der Adipositas" 2014. AWMF Registernummer 050/001. http://www.awmf.org. Accessed 19 June 2017.

55. Deutsche Gesellschaft für Bipolare Störungen (DGBS) e.V. und Deutsche Gesellschaft für Psychiatrie, Psychotherapie und Nervenheilkunde (DGPPN) e.V. S3-Leitlinie zur Diagnostik und Therapie Bipolarer Störungen. Langversion, 2012. AWMF Registernummer: 038-019. http://www.awmf.org. Accessed 19 June 2017.

56. Deutsche Diabetes-Gesellschaft. S3-Leitlinie "Diabetes und Schwangerschaft" 2014. AWMF Registernummer: 057/023. http://www.awmf.org. Accessed 19 June 2017.

57. Deutsche Gesellschaft für Gynäkologie und Geburtshilfe. S3-Leitlinie "Indiaktion und Methodik der Hysterektomie bei benignen Erkrankungen" 2015. AWMF Registernummer: 015/070. http://www.awmf.org. Accessed 26 June 2017.

58. Leitlinienprogramm Onkologie (Deutsche Krebsgesellschaft, Deutsche Krebshilfe, AWMF): S3-Leitlinie Kolorektales Karzinom, Langversion 1.1, 2014. AWMF Registrierungsnummer: 021-007OL. http://leitlinienprogrammonkologie.de/Leitlinien.7.0.html. Accessed 19 June 2017.

59. Deutsche Schmerzgesellschaft. S3 - Leitlinie "Langzeitanwendung von Opioiden bei nicht tumorbedingten Schmerzen - "LONTS" 2015. AWMF Registernummer: 145/003. http://www.awmf.org Accessed 19 June 2017.

60. Leitlinienprogramm Onkologie (Deutsche Krebsgesellschaft, Deutsche Krebshilfe, AWMF): S3-Leitlinie Diagnostik und Therapie der Adenokarzinome des Magens und ösophagogastralen Übergangs, 2012 AWMF Registrierungsnummer: 032-009OL. http://leitlinienprogrammonkologie.de/Leitlinien.7.0.html. Accessed 19 June 2017.

61. Leitlinienprogramm Onkologie (Deutsche Krebsgesellschaft, Deutsche Krebshilfe, AWMF): S3-Leitlinie für die Diagnostik, Therapie und Nachsorge des 
Mammakarzinoms, 2012. AWMF Registrierungsnummer: 032 - 045OL. http:// leitlinienprogramm-onkologie.de/Leitlinien.7.0.html. Accessed 13 July 2017.

62. Leitlinienprogramm Onkologie (Deutsche Krebsgesellschaft, Deutsche Krebshilfe, AWMF): Diagnostik, Therapie und Nachsorge des Melanoms, Langversion 2.0, 2016, AWMF Registernummer: 032/024OL. http:// leitlinienprogramm-onkologie.de/Melanom.65.0.html. Accessed 19 June 2017.

63. Bundesärztekammer (BÄK), Kassenärztliche Bundesvereinigung (KBV), Arbeitsgemeinschaft der Wis-senschaftlichen Medizinischen Fachgesellschaften (AWMF). Nationale VersorgungsLeitlinie Nierenerkrankungen bei Diabetes im Erwachsenenalter - Langfassung, 1. Auflage. Version 6. 2010, zuletzt verändert: September 2015. http://www. dm-nierenerkrankungen.versorgungsleitlinien.de. Accessed 19 June 2017.

64. Leitlinienprogramm Onkologie (Deutsche Krebsgesellschaft, Deutsche Krebshilfe, AWMF): S3-Leitlinie Diagnostik und Therapie der Plattenepithelkarzinome und Adenokarzinome des Ösophagus, Langversion 1.0, 2015, AWMF Registernummer: 021/023OL. http://leitlinienprogrammonkologie.de/Leitlinien.7.0.html. Accessed 19 June 2017.

65. Leitlinienprogramm Onkologie (Deutsche Krebsgesellschaft, Deutsche Krebshiffe, AWMF): Palliativmedizin für Patienten mit einer nicht heilbaren Krebserkrankung, Langversion 1.1, 2015, AWMF-Registernummer: 128/001OL. http:// leitlinienprogrammonkologie.de/Palliativmedizin.80.0.html. Accessed 23 June 2017.

66. AWMF-Institut für Medizinisches Wissensmanagement. S3-Leitlinie "Prophylaxe der venösen Thromboembolie (VTE)" 2015. http://www.awmf. org Accessed 19 June 2017.

67. Deutsche Gesellschaft für Anästhesiologie und Intensivmedizin, et al., S3 Leitlinie "Vermeidung von perioperativer Hypothermie" 2014. AWMF Registernummer: 001/018. http://www.awmf.org Accessed 19 June 2017.

68. van Lieshout J, et al. Consistency of performance indicators for cardiovascular risk management across procedures and panels. Qual Saf Health Care. 2010;19(5):e31.

69. Shekelle PG, et al. Validity of the Agency for Healthcare Research and Quality clinical practice guidelines: how quickly do guidelines become outdated? JAMA. 2001;286(12):1461-7.

70. Marshall MN, et al. Can health care quality indicators be transferred between countries? Qual Saf Health Care. 2003;12(1):8-12.

71. van der Ploeg $\mathrm{E}$, et al. Developing quality indicators for general practice care for vulnerable elders; transfer from US to The Netherlands. Qual Saf Health Care. 2008;17(4):291-5.

72. Petzold T, et al. Quality measurement recommendations relevant to clinical guidelines in Germany and the United Kingdom: (what) can we learn from each other? Inquiry. 2018:55:46958018761495.

73. Bennett $B$, et al. The NICE process for developing quality standards and indicators. Z Evid Fortbild Qual Gesundhwes. 2014;108(8-9):481-6.

74. Alonso-Coello P, et al. GRADE Evidence to Decision (EtD) frameworks: a systematic and transparent approach to making well informed healthcare choices. 2: Clinical practice guidelines. Bmj. 2016;353:i2089.

75. Guyatt G, et al. GRADE guidelines: 1. Introduction-GRADE evidence profiles and summary of findings tables. J Clin Epidemiol. 2011;64(4):383-94.

76. Gill PJ, et al. Primary care quality indicators for children: measuring quality in UK general practice. Br J Gen Pract. 2014;64(629):e752-7.

77. Campbell SM, et al. Framework and indicator testing protocol for developing and piloting quality indicators for the UK quality and outcomes framework. BMC Fam Pract. 2011;12:85.

78. Stelfox HT, Straus SE. Measuring quality of care: considering conceptual approaches to quality indicator development and evaluation. J Clin Epidemiol. 2013;66(12):1328-37.

79. Woitha $\mathrm{K}$, et al. Testing feasibility and reliability of a set of quality indicators to evaluate the organization of palliative care across Europe: a pilot study in 25 countries. Palliat Med. 2015;29(2):157-63.

80. Young GJ, Charns MP, Barbour GL. Quality improvement in the US Veterans Health Administration. Int J Qual Health Care. 1997;9(3):183-8.

81. Johnston A, et al. Systematic reviews of clinical practice guidelines: a methodological guide. J Clin Epidemiol. 2019;108:64-76.

\section{Publisher's Note}

Springer Nature remains neutral with regard to jurisdictional claims in published maps and institutional affiliations.

\section{Ready to submit your research? Choose BMC and benefit from}

- fast, convenient online submission

- thorough peer review by experienced researchers in your field

- rapid publication on acceptance

- support for research data, including large and complex data types

- gold Open Access which fosters wider collaboration and increased citations

- maximum visibility for your research: over $100 \mathrm{M}$ website views per year

At BMC, research is always in progress.

Learn more biomedcentral.com/submissions 